\title{
Upward and Downward Invasion Types of Nasopharyngeal Carcinoma Are Significantly Associated with Survival
}

\author{
Yi Zhuo Li ${ }^{1 *}$, Chuan-Miao Xie ${ }^{1 *}$, Chun-Yan Cuii ${ }^{1}$, Li-Xia Lü ${ }^{2}$, Yao Pan Wu ${ }^{1}$, Rong Zhang ${ }^{1}$, Qing Liu ${ }^{3}$, Wen-Lin \\ Huang ${ }^{4}$, Pei-Hong $\mathrm{Wu}^{1}$ and ${\mathrm{Yi} \mathrm{Li}^{5}}^{5}$ \\ ${ }^{1}$ Imaging Diagnosis and Interventional Center, China \\ ${ }^{2}$ Department of Radiation Oncology, China \\ ${ }^{3}$ Department of Cancer Prevention Research, China \\ ${ }^{4}$ State Key Laboratory of Oncology in Southern China, China \\ ${ }^{5}$ Department of Imaging and Services, Good Samaritan Hospital, NY, USA \\ *Corresponding author: Yi Zhuo Li and Chuan-Miao Xie, Imaging Diagnosis and Interventional Center, State Key Laboratory of \\ Oncology in Southern China, Cancer Center, Sun Yat-sen University, Collaborative Innovation Center for Cancer Medicine. Guangzhou, \\ People's Republic of China
}

\section{ARTICLE INFO}

Received: 业 November 29, 2019

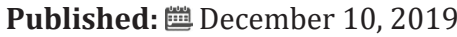

Citation: Yi Zhuo Li, Chuan-Miao Xie, Chun-Yan Cui, Li-Xia Lu, Yao Pan Wu, et al. Upward and Downward Invasion Types of Nasopharyngeal Carcinoma Are Significantly Associated with Survival. Biomed J Sci \& Tech Res 23(5)-2019. BJSTR. MS.ID.003954.

Keywords: Nasopharyngeal Carcinoma; Survival; Invasion Types; Classification; Magnetic Resonance Imaging

Abbreviations: NPC: Nasopharyngeal Carcinoma; MRI: Magnetic Resonance Imaging; AJCC: American Joint Committee on Cancer; TCM: Traditional Chinese Medicine; UICC: Union for International Cancer Control; IMRT: Intensity-Modulated Radiation Therapy; LRFS: Local Relapse-Free Survival

\section{ABSTRACT}

Purpose: Nasopharyngeal Carcinoma (NPC) has distinct TNM stages whose properties and influences on prognosis are not fully understood. This study aimed to analyze the relevant clinical characteristics of NPCs with various invasion types based on Magnetic Resonance Imaging (MRI).

Methods: A total of 817 newly diagnosed NPC patients without distant metastasis were included and underwent MRI. The patients were divided into four groups according to the invaded anatomic region: Local, Upward, Downward, and Extensive. Then, the ages, genders and clinical stage distributions were summarized. The Overall Survival (OS), Distant Metastasis-Free Survival (DMFS), and Local Relapse-Free Survival (LRFS) rates were assessed.

Results: Significant differences were observed between the Upward and Downward groups in gender distribution $(\mathrm{P}=0.020)$ and between the Upward and Extensive groups in age distribution $(\mathrm{P}=0.026)$. The 5-year OS rates of the Local, Upward, Downward and Extensive groups were $90.3 \%, 77.3 \%, 89.0 \%$ and $77.0 \%$, respectively; the DMFS rates were $90.3 \%, 75.4 \%, 89.0 \%$ and $75.7 \%$, respectively; and the LRFS rates were $89.0 \%$, $76.5 \%, 87.7 \%$ and $75.7 \%$, respectively (all $\mathrm{P}<0.001$ ). The Local/Downward and Upward/ Extensive group pairs exhibited similar OS ( $\mathrm{P}=0.517$ and $\mathrm{P}=0.720$, respectively), DMFS $(\mathrm{P}=0.536$ and $\mathrm{P}=0.798$, respectively), and LRFS $(\mathrm{P}=0.589$ and $\mathrm{P}=0.428$, respectively) rates. After adjustments for sex, age, and clinical stage, multivariate analysis indicated that invasion type was an independent prognostic factor for OS (HR=0.509, $\mathrm{P}=0.070)$, DMFS (HR=0.462, $\mathrm{P}=0.038)$ and LRFS (HR=0.559, $\mathrm{P}=0.100)$.

Conclusion: The upward and downward types of NPC are significantly associated with OS, DMFS, and LRFS and are independent prognostic factors for survival. 


\section{Introduction}

Nasopharyngeal Carcinoma (NPC) is the most common malignant neoplasm of the nasopharynx. The World Health Organization classifies NPC into 3 histologic subtypes: keratinizing squamous cell carcinoma (type 1), differentiated non-keratinizing carcinoma (type 2) and undifferentiated non-keratinizing carcinoma (type 3) [1]. Magnetic Resonance Imaging (MRI) is the imaging modality of choice for tumor evaluation and nodal assessment in NPC patients [2-5]. According to the Union for International Cancer Control (UICC) and the American Joint Committee on Cancer (AJCC) TNM staging system [6], tumors that extend into the T3-T4 or N2-N3 structures are classified as advanced-stage (stage III or IV). The US National Comprehensive Cancer Network guidelines recommend that patients with advanced-stage NPC should receive concurrent chemoradiotherapy. However, Mao YP, et al. [7] reported large variations in the clinical outcomes of patients with the same stage who underwent similar treatment regimens. Chen $\mathrm{L}$ et al. demonstrated that adjuvant cisplatin and fluorouracil chemotherapy does not significantly improve failurefree survival after concurrent chemoradiotherapy in locoregionally advanced NPC [8]. These findings suggest that different invasion types of advanced NPC might have various outcomes under current treatment strategies. Therefore, it is important to further define and characterize the various TNM stages to improve prognoses and therapeutic choices. We hypothesized that upward or downward progression, as additional anatomical measures that are widely used in Traditional Chinese Medicine (TCM) [9], might provide valuable information for individualized treatment regimens for advanced-stage NPC patients. Therefore, this study aimed to assess the clinical characteristics of various invasion types of NPC based on MRI. Our findings indicated that upward and downward types of NPC are significantly associated with OS, DMFS, and LRFS.

\section{Material and Methods}

\section{Participants}

This retrospective study was approved by the Institutional Review Board of the unit of the research. Written informed consent was obtained from all patients. Between January 1, 2003 and March 1, 2005, 838 newly diagnosed NPC patients were enrolled. The inclusion criteria were as follows: 1) NPC diagnosis by pathology and no treatment, and 2) nasopharyngeal and neck MRI examination, clinical nasopharyngeal endoscopy, blood examination, and chest and abdominal CT examination pretreatment and during the followup after treatment initiation. Patients with acute inflammation or concurrent head and neck other neoplasms, and distant metastasis were excluded, as were those patients with incomplete clinical data. All lesions were restaged as previously proposed [3].

\section{Imaging Protocols}

All patients underwent MRI on a 1.5-Tesla system (Signa CV/I, GE Healthcare) using the Fast Spin-Echo (FSE) technique. The examined region extended from the suprasellar subarachnoid cistern to the inferior margin of the sternal end of the clavicle. T1-weighted images in the axial, coronal, and sagittal planes (TR range/TE range, 500-600/10-20; number of signals acquired, 2; FOV, $22 \mathrm{~cm}$ ) and T2-weighted FSE images in the axial plane (TR range/TE range, 3000-6000/90-110; number of signals acquired, 2; FOV, 22cm) were obtained. After intravenous injection of gadopentetate dimeglumine (Magnevist, Bayer Schering Pharma), T1-weighted axial, sagittal, and fat-suppressed coronal sequences were sequentially acquired as described previously [10-12].

\section{Image Assessment}

The MR images were independently evaluated by two experienced radiologists, and disagreements were resolved by consensus. The determination of cervical lymph node metastasis was performed according to a previous report [10]. Skull-base invasion was diagnosed as described previously $[13,14]$. The 817 included patients were divided into the following 4 groups: Local (local-regional type; tumors localized within the nasopharynx without upward or downward spread; T1-T2 and N0-N1, Stages I and II ), Upward (upward progressing type; tumors spread upward with skull-base invasion but without downward progression; T3T4 and N0-N1), Downward (downward spread type; tumors spread downward with enlarged neck lymph nodes but without upward spread; T1-T2 and N2-N3), and Extensive (extensive spread type; tumors extensively spread with upward and downward progression; T3-T4 and N2-N3).

\section{Treatment}

All patients were treated with full-course radiation therapy. A total of 597/817 (73.1\%) patients with advanced disease (T3T4 or N2-N3 disease) received neoadjuvant, concomitant, or adjuvant chemotherapy. Patients with MRI-visible cervical lymph nodes received the same high-dose radiation therapy (68-70 Gy). Moreover, 119/817 (14.6\%) patients received Intensity-Modulated Radiation Therapy (IMRT).

\section{Follow-Up}

The followup duration was from treatment initiation to death or the latest examination (quarterly in the first 2 years and every 6 months thereafter). The responses of the tumors and lymph nodes to treatment, including tumor resolution, residual primary tumors, local recurrence, and distant metastases, were assessed by MRI, flexible nasopharyngoscopy, physical examination, and/or biopsy.

\section{Statistical Analysis}

SPSS 18.0 (SPSS, Chicago, IL) was used for statistical analyses. Actuarial rates were calculated by the Kaplan-Meier method [15] and assessed with the log-rank test. The parameters evaluated included the age, gender and clinical stage distributions, as well as the Overall Survival (OS), Distant Metastasis-Free Survival (DMFS), and Local Relapse-Free Survival (LRFS) rates. Multivariate analysis (Cox multi-covariant model) was used to assess the association of invasion type (downward or upward) with survival. Two-tailed $\mathrm{p}<0.05$ was considered statistically significant. 


\section{Results}

\section{Patient Inclusion Flow Chart}

Of the 838 enrolled patients, 21 were excluded (distant metastasis, 17; acute inflammation or concurrent head and neck neoplasms, 2; and incomplete data, 2). The remaining 817 eligible cases were assessed (Figure 1).

\section{Age, Gender and Clinical Stage Distributions}

The Local $(n=155)$, Upward $(n=362)$, Downward $(n=73)$, and Extensive $(n=227)$ groups included $119,260,62$, and 179 males, respectively, and 36,102,11, and 48 females, respectively (Figure 1). The other clinical patient characteristics are summarized in Table 1. The median followup was 69 months (range: 6-110 months). A significant difference was observed between the Upward and Downward groups in gender $(\mathrm{P}=0.020)$ and between the Upward and Extensive groups in age $(\mathrm{P}=0.026$; Table 2). Table 3 outlines the stage distributions of the 4 groups. Significant differences were observed between all groups in the clinical stages $(\mathrm{P}<0.001)$.

Table 1: Clinical characteristics of the 817 NPC patients included in this study.

\begin{tabular}{|c|c|}
\hline Characteristic & No. of patients(\%) \\
\hline Age (years) & \\
\hline$<45$ & $417(51.04)$ \\
\hline$\geq 45$ & $400(48.96)$ \\
\hline Gender & \\
\hline Male & $620(75.89)$ \\
\hline
\end{tabular}

\begin{tabular}{|c|c|}
\hline Female & $197(24.11)$ \\
\hline WHO pathology & $9(1.10)$ \\
\hline Type I & $251(30.72)$ \\
\hline Type II & $557(68.18)$ \\
\hline Type III & \\
\hline T* category $^{*}$ & $57(6.98)$ \\
\hline T1 & $170(20.81)$ \\
\hline T2 & $337(41.25)$ \\
\hline T3 & $253(30.96)$ \\
\hline T4 & $180(22.03)$ \\
\hline N $^{*}$ category & $335(41.00)$ \\
\hline N0 & $255(31.22)$ \\
\hline N1 & $47(5.75)$ \\
\hline N2 & \\
\hline N3 & $29(3.55)$ \\
\hline Clinical stage* & $124(15.18)$ \\
\hline I & $376(46.02)$ \\
\hline II & $288(35.25)$ \\
\hline III & \\
\hline IV & \\
\hline & \\
\hline
\end{tabular}

NPC: nasopharyngeal carcinoma. WHO: World Health Organization.

*According to the 7th UICC/AJCC staging system (UICC: Union for International Cancer Control/AJCC: American Joint Committee on Cancer)? N: node; T: tumor.

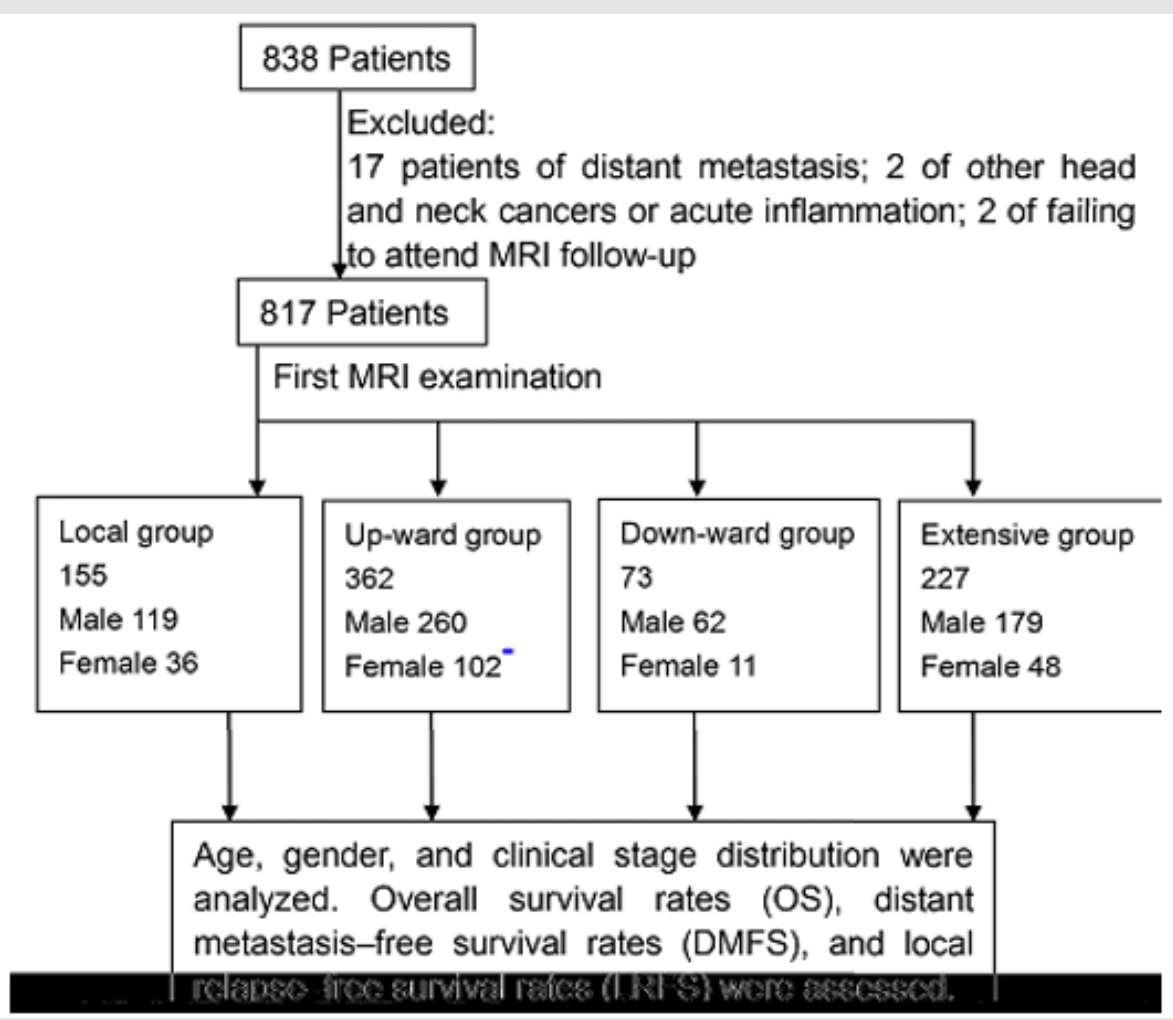

Figure 1: Patient and study flow chart. 
Table 2: Gender, age, and stage distributions of the 817 NPC patients included in this study.

\begin{tabular}{|c|c|c|c|c|c|c|}
\hline & Local Group & Upward Group & Downward Group & Extensive Group & Total & \\
\hline Female (\%) & $36(23.2)$ & $102(28.2)$ & $11(15.1)$ & $48(21.1)$ & 197 & \\
\hline Male (\%) & $119(76.8)$ & $260(71.8)$ & $62(84.9)$ & $179(78.9)$ & 620 & \\
\hline Age $(\bar{x} \pm s) \#$ & $45.04 \pm 11.22$ & $45.73 \pm 11.49$ & $44.88 \pm 10.19$ & $43.56 \pm 11.48$ & & $45.04 \pm 11.22$ \\
\hline \multicolumn{7}{|l|}{ Stage/Group } \\
\hline $\mathrm{I}(\%)$ & $29(18.7)$ & & & $0(00)$ & 29 & \\
\hline II(\%) & $123(79.4)$ & & $1(1.4)$ & $0(0.0)$ & 124 & \\
\hline III(\%) & $1(0.6)$ & $184(50.8)$ & $63(86.3)$ & $128(56.4)$ & 376 & \\
\hline $\mathrm{IVa}(\%)$ & $2(1.3)$ & $177(48.9)$ & $3(4.1)$ & $64(28.2)$ & 246 & \\
\hline $\mathrm{IVb}(\%)$ & $0(0.0)$ & $1(0.3)$ & $6(8.2)$ & $35(15.4)$ & 42 & \\
\hline Total & 155 & 362 & 73 & 227 & 817 & \\
\hline
\end{tabular}

Chi-squared test: $\chi 2=919.72, \mathrm{P}<0.001$.

Table 3: Multivariate analysis of the prognostic factors of NPC patients.

\begin{tabular}{|c|c|c|c|c|}
\hline End point & Factor & P-value & $\operatorname{EXP}(B)$ & 95\% CI for EXP (B) \\
\hline \multirow[t]{12}{*}{ Death } & Sex & & & \\
\hline & Female & & 1 & \\
\hline & Male & 0.048 & 1.764 & $1.007-3.091$ \\
\hline & Age & & & \\
\hline & $<45$ & & 1 & \\
\hline & $\geq 45$ & 0.005 & 1.353 & $1.098-1.670$ \\
\hline & Stage & & & \\
\hline & $\mathrm{I}+\mathrm{II}$ & & 1 & \\
\hline & III+IV & 0.001 & 1.967 & $1.323-2.921$ \\
\hline & Group & & & \\
\hline & U-ward & & 1 & \\
\hline & D-ward & 0.07 & 0.509 & $0.245-1.057$ \\
\hline \multirow[t]{12}{*}{ Distant failure } & Sex & & & \\
\hline & Female & & 1 & \\
\hline & Male & 0.034 & 1.18 & $1.046-3.099$ \\
\hline & Age & & & \\
\hline & $<45$ & & 1 & \\
\hline & $\geq 45$ & 0.003 & 1.359 & $1.110-1.662$ \\
\hline & Stage & & & \\
\hline & $\mathrm{I}+\mathrm{II}$ & & 1 & \\
\hline & III+IV & 0 & 1.979 & $1.351-2.901$ \\
\hline & Group & & & \\
\hline & U-ward & & 1 & \\
\hline & D-ward & 0.038 & 0.462 & $0.223-0.958$ \\
\hline \multirow[t]{7}{*}{ Local failure } & Sex & & & \\
\hline & Female & & 1 & \\
\hline & Male & 0.055 & 1.706 & $0.989-2.941$ \\
\hline & Age & & & \\
\hline & $<45$ & & 1 & \\
\hline & $\geq 45$ & 0.004 & 1.353 & $1.101-1.662$ \\
\hline & Stage & . & & \\
\hline
\end{tabular}




\begin{tabular}{|l|c|c|c|c|}
\hline & I+II & & 1 & 2.024 \\
\hline & III+IV & 0 & & $1.376-2.978$ \\
\hline & Group & & 1 & \\
\hline & U-ward & & 0.559 & $0.280-1.116$ \\
\hline
\end{tabular}

\section{Survival in Local, Upward, Downward and Extensive groups}

For the whole population, the 5-year OS, DMFS, and LRFS rates were $80.8 \%, 79.5 \%$ and $79.6 \%$, respectively. The 5 -year OS rates of the Local, Upward, Downward and Extensive groups were $90.3 \%$, $77.3 \%, 89.0 \%$ and $77.0 \%$, respectively, and there was a significant difference between these groups $(\mathrm{P}<0.001$, Figure 2a). There was a significant difference between the Upward and Downward groups $(\mathrm{P}<0.001)$ but not between the Local and Downward groups $(\mathrm{P}=0.517)$ or between the Upward and Extensive groups $(\mathrm{P}=0.720)$. The 5-year DMFS rates were $90.3 \%, 75.4 \%, 89.0 \%$ and $75.7 \%$ for the
Local, Upward, Downward and Extensive groups, respectively, and there was a significant difference $(\mathrm{P}<0.001$, Figure $2 \mathrm{~b})$. A significant difference was found between the Upward and Downward groups $(\mathrm{P}<0.001)$. The Local and Downward groups $(\mathrm{P}=0.536)$ and Upward and Extensive groups $(\mathrm{P}=0.798)$ exhibited similar vales. The 5-year LRFS rates were $89.0 \%, 76.5 \%, 87.7 \%$ and $75.7 \%$ in the Local, Upward, Downward and Extensive groups, respectively, and there was a significant difference $(\mathrm{P}<0.001$, Figure $2 \mathrm{c})$. There was a significant difference between the Upward and Downward groups $(\mathrm{P}<0.001)$. The Local and Downward groups $(\mathrm{P}=0.589)$ and Upward and Extensive groups $(\mathrm{P}=0.428)$ exhibited similar values.

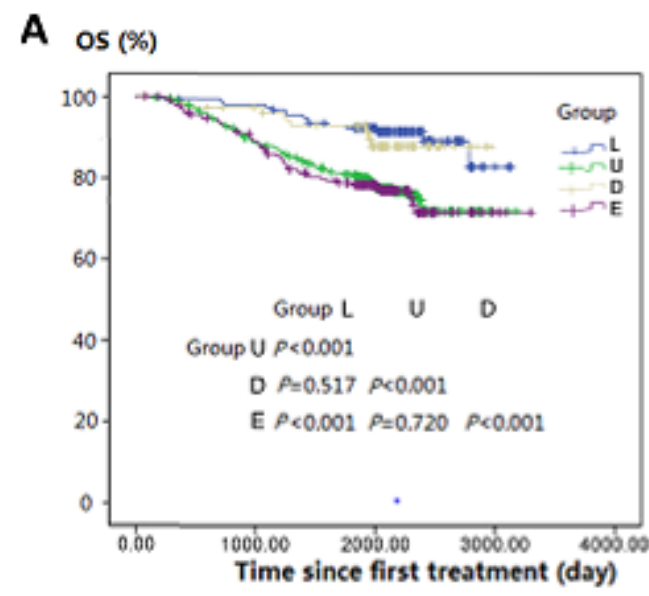

\section{$B_{\text {DMFS (*) }}$}

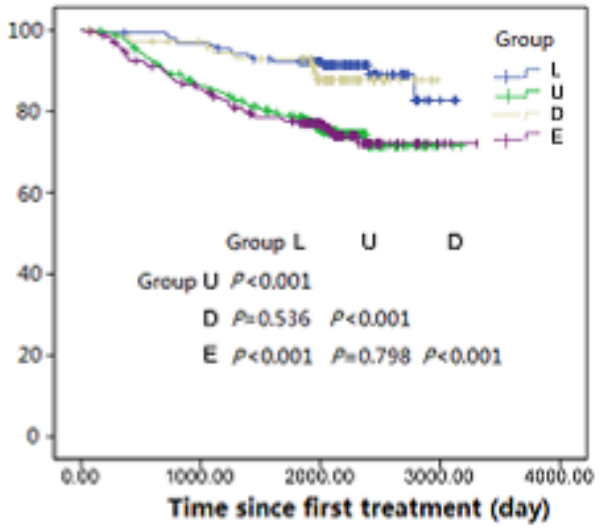

\section{LRFS (\%)}

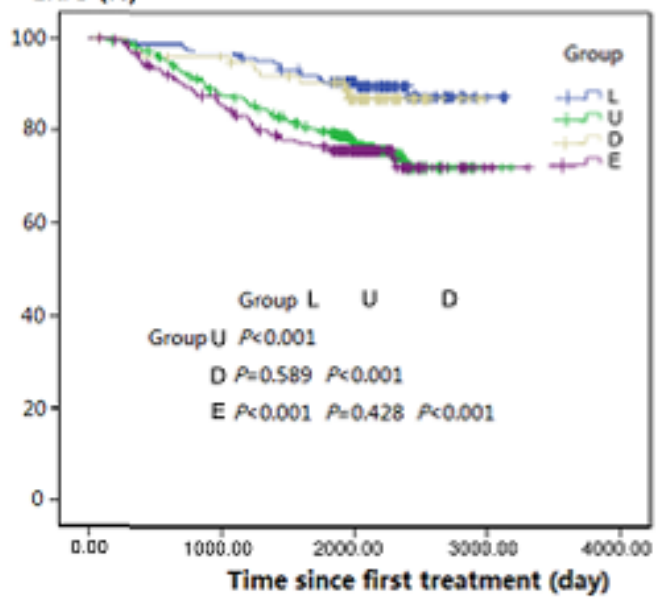

Figure 2: Patient prognoses based on upward or downward progression types.

A. Kaplan-Meier curves for Overall Survival (OS),

B. Distant Metastasis-Free Survival (DMFS), and

C. Local Relapse-Free Survival (LRFS) for the 817 patients included in this study. 
Comparison of the Upward and Downward groups among stage III patients

The OS was slightly lower in the Upward group than the Downward group, but the difference was not significant $(\chi 2=3.375$,
$\mathrm{P}=0.066$, Figure 3a). The DMFS $(\chi 2=3.954, \mathrm{P}=0.047)$ and LRFS $(\chi 2=3.921, \mathrm{P}=0.048)$ were significantly higher in the Downward group than in the Upward group (Figure $3 b$ \& 3c).

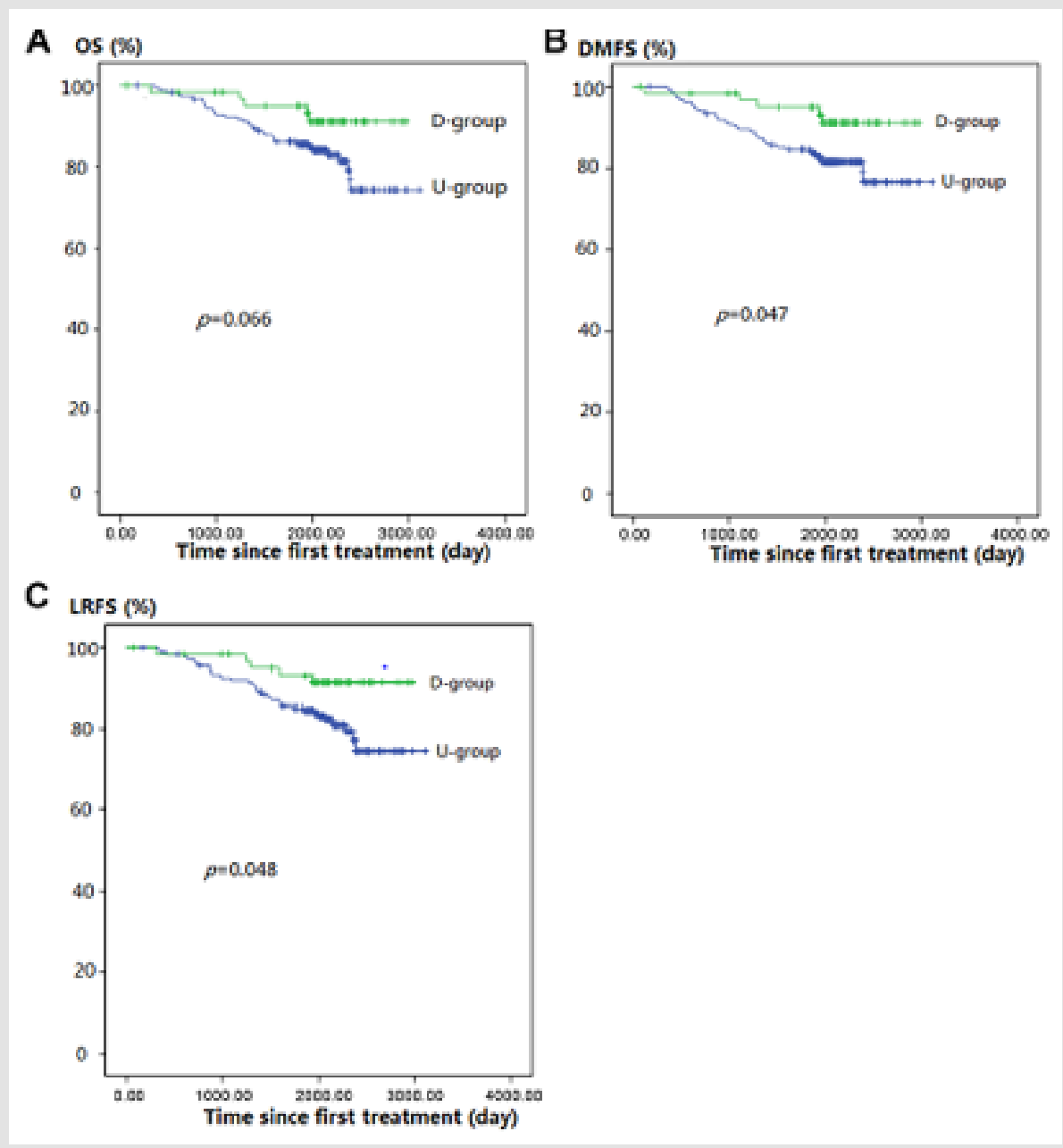

Figure 3: Patient prognoses based on upward or downward progression types among stage III patients.

A. Kaplan-Meier curves for overall survival (OS),

B. Distant metastasis-free survival (DMFS) and

C. Local Relapse-Free Survival (LRFS) for the 817 patients included in this study.

\section{Invasion Type Is an Independent Risk Factor for Survival}

After adjustments for sex, age, and clinical stage, invasion type was found to be an independent prognostic factor for OS (HR=0.509, $\mathrm{p}=0.070)$, DMFS (HR=0.462, $\mathrm{p}=0.038)$ and LRFS (HR=0.559, $\mathrm{p}=0.100$ ) (Table 3).

\section{Discussion}

Several prognostic factors for NPC have been proposed, including T classification and other clinical and molecular prognostic variables [16]. The gross tumor volume of the nasopharynx is considered a good prognostic factor [17-19]. Lu LX et al. [20] demonstrated that combining tumor volume and pretreatment EBV DNA level further improves the accuracy of prognostic evaluation. Ou Yang PY et al.
[21] evaluated the body mass index and survival of NPC patients by propensity-matched analysis. Several molecular biomarkers for NPC diagnosis and prognosis have been investigated, including tissue- and blood-derived factors [22]. For example, the expression of two tissue-based microRNAs is associated with survival and distant metastasis in NPC $[9,23]$. Other circulating biomarkers have been identified in patients with NPC. These include EBV-encoded factors, such as LMP1 and Bam H1 reading frame 1 proteins [24], and noncoding RNA molecules, such as EBER1, EBER2, and BART microRNAs $[25,26]$.

However, further validation is required before clinical implementation. Moreover, the perfusion fraction and pseudodiffusion coefficient have been demonstrated to be independent 
predictors of T staging, while pure diffusion independently predicts $\mathrm{N}$ staging in NPC [27]. In addition, quantitative dynamic contrastenhanced Magnetic Resonance Imaging (DCE-MRI) parameters are significantly related to clinical stage in NPC [28]. Although much has been proposed regarding NPC development and progression, much remains unclear [22]. Only two Chinese studies have assessed the upward and downward spreading types of NPC. Liang WJ et al. [29] proposed that DIO2 gene expression is higher in the downward spread type than in its upward counterpart and may be closely related to metastasis in NPC. Moreover, Mo LG et al. [30] demonstrated that cervical lymph node metastasis is a critical factor of distant metastasis in NPC. The current study evaluated the associations of tumor spread type and outcomes from another perspective.

As demonstrated above, most advanced NPC patients (stages III or IV) displayed the upward spread type (362/664, 54.52\%). Significant differences were observed between the Upward and Downward groups in the five-year OS, DMFS and LRFS rates. The Upward group had poorer prognoses that were similar to those of the Extensive group. Moreover, the prognoses in the Downward group (including advanced-stage patients) were more favorable and similar to those of the Local group (stage I or II) patients (Figure 2). No significant differences were found between the upward and extensive types or between the downward and local-regional types. These findings revealed that the upward and extensive spread types have similar prognoses as do the downward and local-regional types. Therefore, the use of upward or downward progression may further help to achieve more appropriate prognoses and design individualized treatments when combined with existing staging systems. In this study, the clinical staging of NPC patients depended only on the T-classification and not on the $\mathrm{N}$-classification, and the findings are inconsistent with the current AJCC/UICC tumor staging system for NPC. This inconsistency is likely because the treatment modalities for NPC have greatly advanced.

The aim of the AJCC/UICC staging system for NPC is to guide prognosis, propose treatment planning, and assess outcomes while providing the convenience of data sharing between various clinical and research centers. With advances in diagnosis and treatment strategies, the staging systems for malignant tumors should be revised and improved. Hence, for consistency with hazard consistency, hazard discrimination, and prognosis prediction, we propose that NPC classification might include upward or downward progression as an additional metric. The current retrospective study has several limitations. First, the grouping was based on the invasion types according to MR imaging, which contrasts with the previous literature. Therefore, this method should be validated. Second, the use of 5-mm-thick slices in MRI could lead to omitted information. Finally, advanced NPC patients underwent different treatment modalities, which might have affected the treatment outcomes.

\section{Conclusion}

In conclusion, there were more female patients in the Upward group than in the Downward group. The patient age in the Upward group was slightly elevated compared with that in the Downward and Extensive groups. Different invasion types of NPC were significantly associated with OS, DMFS, and LRFS. The prognosis of the Upward group was poorer than that of the Downward group. The current findings suggested that each invasion type of advanced NPC probably exhibits an individual biological heterogeneity. We propose to include invasion type in a comprehensive algorithm for NPC patient classification. This process might help to identify proper treatment regimens and improve the hazard discrimination and consistency of the current AJCC/UICC staging system in the next revision of NPC.

\section{References}

1. Shanmugaratnam K (1978) Histological typing of nasopharyngeal carcinoma. IARC Sci Publ pp. 3-12.

2. Chan M, Sahgal A, Fatterpekar G (2014) Imaging of nasopharyngeal carcinoma. J Nasopharyng Carcinoma 1: e11.

3. King AD, Vlantis AC, Bhatia KS, Chan AT, Ahuja AT, et al. (2011) Primary nasopharyngeal carcinoma: diagnostic accuracy of MR imaging versus that of endoscopy and endoscopic biopsy. Radiology 258: 531-537.

4. King AD, Vlantis AC, Tsang RK, Ahuja AT, Lui HK, et al. (2006) Magnetic resonance imaging for the detection of nasopharyngeal carcinoma. AJNR Am J Neuroradiol 27: 1288-1291.

5. Yu E, O'Sullivan B, Kim J, Siu L, Bartlett E (2010) Magnetic resonance imaging of nasopharyngeal carcinoma. Expert Rev Anticancer Ther 10(3): 365-375.

6. Edge SB, Compton CC (2010) The American Joint Committee on Cancer: the 7th edition of the AJCC cancer staging manual and the future of TNM. Ann Surg Oncol 17: 1471-1474.

7. Mao YP, Xie FY, Liu LZ, Liu MZ, Ma J, et al. (2009) Re-evaluation of 6th edition of AJCC staging system for nasopharyngeal carcinoma and proposed improvement based on magnetic resonance imaging. Int J Radiat Oncol Biol Phys 73(5): 1326-1334.

8. Chen L, Hu CS, Chen XZ, Liu MZ, Ma J, et al. (2012) Concurrent chemoradiotherapy plus adjuvant chemotherapy versus concurrent chemoradiotherapy alone in patients with locoregionally advanced nasopharyngeal carcinoma: A phase 3 multicentre randomised controlled trial. Lancet Oncol 13(2): 163-171.

9. Liu N, Chen NY, Cui RX, Wang HY, Ma J, et al. (2012) Prognostic value of a microRNA signature in nasopharyngeal carcinoma: a microRNA expression analysis. Lancet Oncol 13: 633-641.

10. Zhang GY, Liu LZ, Wei WH, Li YZ, Liu XW, et al. (2010) Radiologic criteria of retropharyngeal lymph node metastasis in nasopharyngeal carcinoma treated with radiation therapy. Radiology 255: 605-612.

11. Li YZ, Cai PQ, Xie CM, Zhong R, Wu PH, et al. (2013) Nasopharyngeal cancer: impact of skull base invasion on patients prognosis and its potential implications on TNM staging. Eur J Radiol 82: e107-e111.

12. Li YZ, Xie CM, Wu YP, Lu CY, Wu PH, et al. (2015) Nasopharyngeal carcinoma patients with retropharyngeal lymph node metastases: a minimum axial diameter of $6 \mathrm{~mm}$ is a more accurate prognostic predictor than $5 \mathrm{~mm}$. AJR Am J Roentgenol 204: 20-23.

13. Chong VF, Fan YF (1996) Skull base erosion in nasopharyngeal carcinoma: detection by CT and MRI. Clin Radiol 51: 625-631. 
14. Nishioka T, Shirato H, Kagei K (2000) Skull-base invasion of nasopharyngeal carcinoma: magnetic resonance imaging findings and therapeutic implications. Int J Radiat Oncol Biol Phys 47: 395-400.

15. Kaplan EL, Meier P (1958) Nonparametric Estimation from Incomplete Observations. J Am Stat Assoc 53: 457-481.

16. Chua ML, Wee JT, Hui EP, Chan AT (2016) Nasopharyngeal carcinoma. Lancet 387: 1012-1024.

17. Lee CC, Huang TT, Lee MS (2010) Clinical application of tumor volume in advanced nasopharyngeal carcinoma to predict outcome. Radiat Oncol 5: 20 .

18. Wu Z, Zeng RF, Su Y (2013) Prognostic significance of tumor volume in patients with nasopharyngeal carcinoma undergoing intensitymodulated radiation therapy. Head Neck 35(5): 689-694.

19. Qin L, Wu F, Lu H, Li G, Wang R, et al. (2016) Tumor Volume Predicts Survival Rate of Advanced Nasopharyngeal Carcinoma Treated with Concurrent Chemoradiotherapy. Otolaryngol Head Neck Surg 155: 598605.

20. Lu L, Li J, Zhao C, Jia W, Lu T, et al. (2016) Prognostic efficacy of combining tumor volume with Epstein-Barr virus DNA in patients treated with intensity-modulated radiotherapy for nasopharyngeal carcinoma. Oral Oncol 60(1): 18-24.

21. OuYang PY, Zhang LN, Tang J (2016) Evaluation of Body Mass Index and Survival of Nasopharyngeal Carcinoma by Propensity-Matched Analysis: An Observational Case-Control Study. Medicine (Baltimore) 95: e2380.

22. Bruce JP, Yip K, Bratman SV (2015) Nasopharyngeal Cancer: Molecular Landscape. J Clin Oncol 33: 3346-3355.

23. Bruce JP, Hui AB, Shi W (2015) Identification of a microRNA signature associated with risk of distant metastasis in nasopharyngeal carcinoma. Oncotarget 6: 4537-4550.

ISSN: 2574-1241

DOI: 10.26717/BJSTR.2019.23.003954

Yi Zhuo Li, Chuan-Miao Xie. Biomed J Sci \& Tech Res

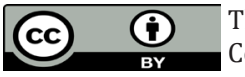

This work is licensed under Creative Commons Attribution 4.0 License

Submission Link: https://biomedres.us/submit-manuscript.php
24. Houali K, Wang X, Shimizu Y, Bouguermouh A, Ooka T, et al. (2007) A new diagnostic marker for secreted Epstein-Barr virus encoded LMP1 and BARF1 oncoproteins in the serum and saliva of patients with nasopharyngeal carcinoma. Clin Cancer Res 13: 4993-5000.

25. Zhang G, Zong J, Lin S, Pan J, Chen H, et al. (2015) Circulating EpsteinBarr virus microRNAs miR-BART7 and miR-BART13 as biomarkers for nasopharyngeal carcinoma diagnosis and treatment. Int J Cancer 136: e301-e312.

26. Lo KW, Lo YM, Leung SF, Lee JC, Huang DP, et al. (1999) Analysis of cellfree Epstein-Barr virus associated RNA in the plasma of patients with nasopharyngeal carcinoma. Clin Chem 45: 1292-1294.

27. Xiao Y, Pan J, Chen Y, He Z, Zheng X, et al. (2015) Intravoxel Incoherent Motion-Magnetic Resonance Imaging as an Early Predictor of Treatment Response to Neoadjuvant Chemotherapy in Locoregionally Advanced Nasopharyngeal Carcinoma. Medicine (Baltimore) 94(24): e973.

28. Huang B, Wong CS, Whitcher B, Chan Q Khong PL, et al. (2013) Dynamic contrast-enhanced magnetic resonance imaging for characterising nasopharyngeal carcinoma: Comparison of semiquantitative and quantitative parameters and correlation with tumour stage. Eur Radiol 23: $1495-1502$.

29. Liang WJ, Qiu F, Hong MH, Min HQ Zeng YX, et al. (2008) Differentially expressed genes between upward and downward progressing types of nasopharyngeal carcinoma. Ai Zheng 27(5): 460-465.

30. Mo L, Weng J, Zeng F, Li Z, Kuang G, et al. (2010) The relationship between extend types and distant metastasis of nasopharyngeal carcinoma. Lin Chung Er Bi Yan Hou Tou Jing Wai Ke Za Zhi 24(12): 554-555.

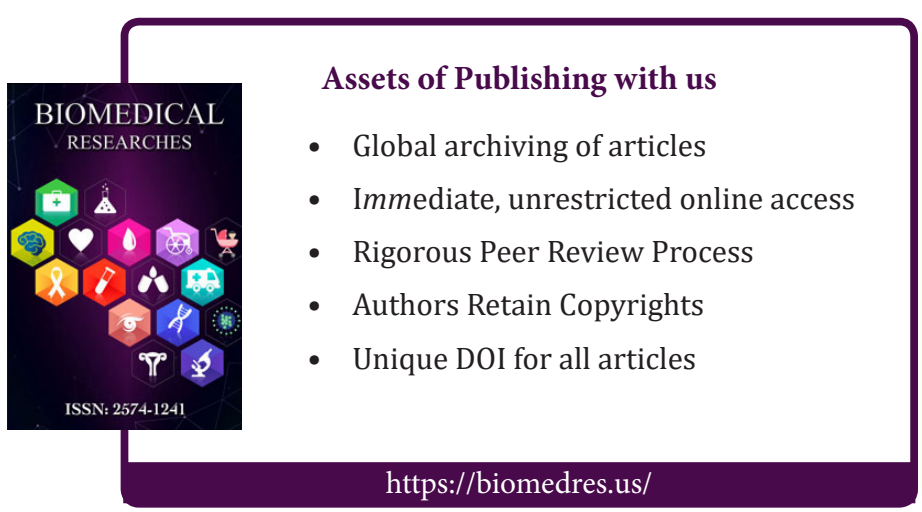

